\title{
Entanglement and nonlocality of one- and two-mode combination squeezed state*
}

\author{
Li-yun $\mathrm{Hu}^{1 \dagger}$, Xue-xiang $\mathrm{Xu}^{1,2}$, Qin Guo ${ }^{1}$, and Hong-yi Fan ${ }^{2}$ \\ ${ }^{1}$ College of Physics \& Communication Electronics, Jiangxi Normal University, Nanchang 330022, China \\ ${ }^{2}$ Department of Physics, Shanghai Jiao Tong University, Shanghai 200030, China
}

October 16, 2018

\begin{abstract}
We investigate the entanglement and nonlocality properties of one- and two-mode combination squeezed vacuum state (OTCSS, with two-parameter $\lambda$ and $\gamma$ ) by analyzing the logarithmic negativity and the Bell's inequality. It is found that this state exhibits larger entanglement than that of the usual two-mode squeezed vacuum state (TSVS), and that in a certain regime of $\lambda$, the violation of Bell's inequality becomes more obvious, which indicates that the nonlocality of OTCSS can be stronger than that of TSVS. As an application of OTCSS, the quantum teleportaion is examined, which shows that there is a region spanned by $\lambda$ and $\gamma$ in which the fidelity of OTCSS channel is larger than that of TSVS.
\end{abstract}

Keywords: Entanglement, nonlocality, IWOP technique, teleportation

PACS number(s): 42.50.Dv, 03.65.Wj, 03.67.Mn

\section{Introduction}

Entanglement between quantum systems plays a key role in quantum information processing, such as quantum teleportation, dense coding, and quantum cloning. In recent years, various entangled states have brought considerable attention and interests of physicists because of their potential uses in quantum communication [1,2]. For instance, the two-mode squeezed state is a typical entangled state of continuous variable and exhibits quantum entanglement between the idle-mode and the signal-mode in a frequency domain manifestly. Theoretically, the two-mode squeezed state is constructed by the two-mode squeezing operator $S=\exp \left[\lambda\left(a_{1} a_{2}-a_{1}^{\dagger} a_{2}^{\dagger}\right)\right][3-5]$ acting on the two-mode vacuum state $|00\rangle$,

$$
S|00\rangle=\operatorname{sech} \lambda \exp \left[-a_{1}^{\dagger} a_{2}^{\dagger} \tanh \lambda\right]|00\rangle,
$$

where $\lambda$ is a squeezing parameter, the disentangling of $S$ can be obtained by using $\mathrm{SU}(1,1)$ Lie algebra, $\left[a_{1} a_{2}, a_{1}^{\dagger} a_{2}^{\dagger}\right]=a_{1}^{\dagger} a_{1}+a_{2}^{\dagger} a_{2}+1$, or by using the entangled state representation

${ }^{*}$ Work supported by a grant from the Key Programs Foundation of Ministry of Education of China (No. 210115) and the Research Foundation of the Education Department of Jiangxi Province of China (No. GJJ10097).

${ }^{\dagger}$ Corresponding author. E-mail: hlyun2008@126.com. 
$|\eta\rangle$ [6] 8], which was constructed according to the idea of Einstein, Podolsky and Rosen in their argument that quantum mechanics is incomplete [9].

Using the relation between Bosonic operators and the coordinate $Q_{i}$, momentum $P_{i}, Q_{i}=$ $\left(a_{i}+a_{i}^{\dagger}\right) / \sqrt{2}, P_{i}=\left(a_{i}-a_{i}^{\dagger}\right) /(\sqrt{2} \mathrm{i})$, and introducing the two-mode quadrature operators of light field, $x_{1}=\left(Q_{1}+Q_{2}\right) / 2, x_{2}=\left(P_{1}+P_{2}\right) / 2$, the variances of $x_{1}$ and $x_{2}$ in the state $S|00\rangle$ are in the standard form

$$
\left\langle 00\left|S^{\dagger} x_{2}^{2} S\right| 00\right\rangle=\frac{1}{4} e^{-2 \lambda},\left\langle 00\left|S^{\dagger} x_{1}^{2} S\right| 00\right\rangle=\frac{1}{4} e^{2 \lambda},
$$

thus we get the standard squeezing for the two quadrature: $x_{1} \rightarrow \frac{1}{2} e^{\lambda} x_{1}, x_{2} \rightarrow \frac{1}{2} e^{-\lambda} x_{2}$. On the other hand, the two-mode squeezing operator can also be recast into the form $S=\exp \left[i \lambda\left(Q_{1} P_{2}+Q_{2} P_{1}\right)\right]$. Then some interesting questions naturally rise: what is the property of the following operator

$$
V=\exp \left[-\mathrm{i}\left(\lambda_{1} Q_{1} P_{2}+\lambda_{2} Q_{2} P_{1}\right)\right]
$$

with two parameters $\lambda_{1}=\lambda e^{\gamma}, \lambda_{2}=\lambda e^{-\gamma}, \lambda>0$ ? What is the normally ordered expansion of $V$ and what is the state $V|00\rangle$ ? What are the entanglement and nonlocality properties of $V|00\rangle$ ? When $\gamma=0$, Eq.(3) just reduces to the usual two-mode squeezing operator $S$. Thus we can consider $V$ as a generalized two-mode squeezing operator and $V|00\rangle$ as oneand two-mode combination squeezed vacuum state (OTCSS).

In this paper, we investigate entanglement properties and quantum nonlocality of $V|00\rangle$ in terms of logarithmic negativity and the Bell's inequality, respectively. Subsequently, we consider its application in the field of quantum teleportation by using the characteristicfunction formula. It is shown that this state exhibits larger entanglement than that of the usual two-mode squeezed vacuum state (TSVS); and in a certain smaller regime of $\lambda$, that the nonlocality of this state can be stronger than that of TSVS due to the presence of $\gamma$. In addition, application to quantum teleportation with OTCSS is also considered, which shows that there is a region spanned by $\lambda$ and $\gamma$ in which the fidelity of OTCSS channel is larger than that of TSVS.

Our paper is arranged as follows. In section 2, we derive the normal ordering form of one- and two-mode combination squeezing operator by using the technique of integration within an ordered product (IWOP) of operators. In section 3, using the Weyl ordering form of single-mode Wigner operator and the order-invariance of Weyl ordered operators under similar transformations, we derive analytically the Wigner function of $V|00\rangle$. Sections 4 and 5 are devoted to investigating the entanglement properties and the nonlocal properties OTCSS by using the Bell's inequality and the logarithmic negativity, respectively. An application to quantum teleportation with OTCSS is involved in section 6 . We end with the main conclusions of our work.

\section{The normal ordering form of $V$ and fluctuations in $V|00\rangle$}

In order to know $V|00\rangle$, we need to derive the normal ordering form of the unitary operator $V$ by virtue of the IWOP technique [10 12]. Using the Baker-Hausdorff formula,

$$
e^{A} B e^{-A}=B+[A, B]+\frac{1}{2 !}[A,[A, B]]+\frac{1}{3 !}[A,[A,[A, B]]]+\cdots
$$


and noticing that

$$
\begin{aligned}
i\left[\lambda_{1} Q_{1} P_{2}+\lambda_{2} Q_{2} P_{1}, Q_{1}\right] & =\lambda_{2} Q_{2}, \\
i\left[\lambda_{1} Q_{1} P_{2}+\lambda_{2} Q_{2} P_{1}, Q_{2}\right] & =\lambda_{1} Q_{1}, \\
i\left[\lambda_{1} Q_{1} P_{2}+\lambda_{2} Q_{2} P_{1}, P_{1}\right] & =-\lambda_{1} P_{2}, \\
i\left[\lambda_{1} Q_{1} P_{2}+\lambda_{2} Q_{2} P_{1}, P_{2}\right] & =-\lambda_{2} P_{1},
\end{aligned}
$$

we have

$$
\begin{aligned}
& V^{-1} Q_{1} V=Q_{1} \cosh \lambda+Q_{2} e^{-\gamma} \sinh \lambda, \\
& V^{-1} Q_{2} V=Q_{2} \cosh \lambda+Q_{1} e^{\gamma} \sinh \lambda, \\
& V^{-1} P_{1} V=P_{1} \cosh \lambda-P_{2} e^{\gamma} \sinh \lambda, \\
& V^{-1} P_{2} V=P_{2} \cosh \lambda-P_{1} e^{-\gamma} \sinh \lambda .
\end{aligned}
$$

Thus, in order to keep the eigenvalues invariant under the $V$ transformation, i..e.,

$$
V^{-1} Q_{k} V\left|q_{1} q_{2}\right\rangle^{\prime}=q_{k}\left|q_{1} q_{2}\right\rangle^{\prime},(k=1,2),
$$

the base vector must be changed to

$$
\left|q_{1} q_{2}\right\rangle^{\prime}=V^{-1}\left|q_{1} q_{2}\right\rangle=\left|\Lambda^{-1}\left(\begin{array}{c}
q_{1} \\
q_{2}
\end{array}\right)\right\rangle, \Lambda=\left(\begin{array}{cc}
\cosh \lambda & e^{-\gamma} \sinh \lambda \\
e^{\gamma} \sinh \lambda & \cosh \lambda
\end{array}\right),
$$

where $\left|q_{1} q_{2}\right\rangle=\left|q_{1}\right\rangle \otimes\left|q_{2}\right\rangle$, and $\left|q_{k}\right\rangle$ is the coordinate eigenstate,

$$
\left|q_{k}\right\rangle=\pi^{-1 / 4} \exp \left[-\frac{1}{2} q^{2}+\sqrt{2} q a^{\dagger}-\frac{1}{2} a^{\dagger 2}\right]|0\rangle .
$$

Using the completeness raltion $\int_{-\infty}^{\infty} d q_{1} d q_{2}\left|q_{1}, q_{2}\right\rangle\left\langle q_{1}, q_{2}\right|=1$, we have

$$
V^{-1}=\int_{-\infty}^{\infty} d q_{1} d q_{2}\left|\Lambda^{-1}\left(\begin{array}{l}
q_{1} \\
q_{2}
\end{array}\right)\right\rangle\left\langle q_{1}, q_{2}\right|
$$

which leads to

$$
V=\int_{-\infty}^{\infty} d q_{1} d q_{2}\left|\Lambda\left(\begin{array}{c}
q_{1} \\
q_{2}
\end{array}\right)\right\rangle\left\langle q_{1}, q_{2}\right|
$$

Actually, one can check (17) by $V^{-1} V=V V^{-1}=1$. Further using the vacuum projector $|00\rangle\langle 00|=: \exp \left[-a^{\dagger} a-b^{\dagger} b\right]:(:$ : denoting normal ordering), as well as the IWOP technique, we can put $V$ into the normal ordering form [13],

$$
\begin{aligned}
V & =\frac{2}{\sqrt{L}} \exp \left\{\frac{1}{L}\left[\left(b^{\dagger 2}-a^{\dagger 2}\right) \sinh ^{2} \lambda \sinh 2 \gamma+2 a^{\dagger} b^{\dagger} \sinh 2 \lambda \cosh \gamma\right]\right\} \\
& : \exp \left\{\frac{4}{L}\left[\left(a^{\dagger} a+b^{\dagger} b\right) \cosh \lambda+\left(b^{\dagger} a-a^{\dagger} b\right) \sinh \lambda \sinh \gamma\right]-a^{\dagger} a-b^{\dagger} b\right\}: \\
& \exp \left\{\frac{1}{L}\left[\left(b^{2}-a^{2}\right) \sinh ^{2} \lambda \sinh 2 \gamma-2 a^{\dagger} b^{\dagger} \sinh 2 \lambda \cosh \gamma\right]\right\},
\end{aligned}
$$

where $L=4\left(1+\sinh ^{2} \gamma \tanh ^{2} \lambda\right) \cosh ^{2} \lambda$. Eq. (18) is just the normal ordering form of $V$. It is obviously to see that when $\gamma=0$, Eq.(18) just reduces to the usual two-mode squeezing 
operator. Operating $V$ on the two-mode vacuum state $|00\rangle$, we obtain the squeezed vacuum state,

$$
V|00\rangle=\frac{2}{\sqrt{L}} \exp \left\{\frac{1}{L}\left[\left(b^{\dagger 2}-a^{\dagger 2}\right) \sinh ^{2} \lambda \sinh 2 \gamma+2 a^{\dagger} b^{\dagger} \sinh 2 \lambda \cosh \gamma\right]\right\}|00\rangle .
$$

On the other hand, by using the transformations Eqs.(9)-(12), one can derive the variances of $x_{1}$ and $x_{2}$ in the state $V|00\rangle[13$,

$$
\begin{aligned}
& \left\langle\left(\Delta x_{1}\right)^{2}\right\rangle=\frac{1}{4}\left(\cosh 2 \lambda+2 \sinh ^{2} \lambda \sinh ^{2} \gamma+\sinh 2 \lambda \cosh \gamma\right), \\
& \left\langle\left(\Delta x_{2}\right)^{2}\right\rangle=\frac{1}{4}\left(\cosh 2 \lambda+2 \sinh ^{2} \lambda \sinh ^{2} \gamma-\sinh 2 \lambda \cosh \gamma\right),
\end{aligned}
$$

which indicate that the variances are not only dependent on parameter $\lambda$, but also on parameter $\gamma$. When $\gamma=0$, Eqs.(20) and (21) reduce to $\left\langle\left(\Delta x_{1}\right)^{2}\right\rangle=\frac{1}{4} e^{2 \lambda}$, and $\left\langle\left(\Delta x_{2}\right)^{2}\right\rangle=$ $\frac{1}{4} e^{-2 \lambda}$, corresponding to the usual TSVS. In particular, by modulating the two parameters $(\lambda$ and $\gamma)$, we can realize that

$$
\left\langle\left(\Delta x_{1}\right)^{2}\right\rangle>\frac{1}{4} e^{2 \lambda},\left\langle\left(\Delta x_{2}\right)^{2}\right\rangle<\frac{1}{4} e^{-2 \lambda}
$$

whose condition is given by

$$
0<\tanh \lambda<\frac{1}{1+\cosh \gamma}, \lambda>0
$$

which mean that the OTCSS may exhibit stronger squeezing in one quadrature than that of the TSVS while exhibiting weaker squeezing in another quadrature when the condition (23) satisfied. Then, can the OTCSS exhibits stronger nonlocality or more observable violation of Bell's inequality? In the following, we pay our attention to these two aspects.

\section{Wigner function of $V|00\rangle$}

Wigner distribution functions [14 16] of quantum states are widely studied in quantum statistics and quantum optics. Now we derive the expression of the Wigner function of $V|00\rangle$. Here we take a new method to do it. Recalling that in Ref. [17 19] we have introduced the Weyl ordering form of single-mode Wigner operator $\Delta_{1}\left(q_{1}, p_{1}\right)$,

$$
\Delta_{1}\left(q_{1}, p_{1}\right)=: \delta\left(q_{1}-Q_{1}\right) \delta\left(p_{1}-P_{1}\right):
$$

its normal ordering form is

$$
\Delta_{1}\left(q_{1}, p_{1}\right)=\frac{1}{\pi}: \exp \left[-\left(q_{1}-Q_{1}\right)^{2}-\left(p_{1}-P_{1}\right)^{2}\right]:,
$$

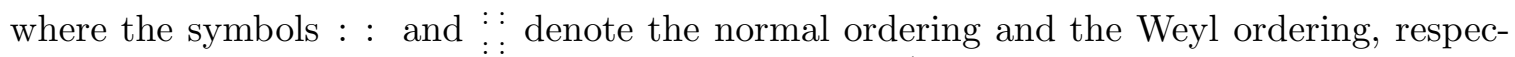
tively. Note that the order of Bose operators $a_{1}$ and $a_{1}^{\dagger}$ within a normally ordered product and a Weyl ordered product can be permuted. That is to say, even though $\left[a_{1}, a_{1}^{\dagger}\right]=1$, we can have $: a_{1} a_{1}^{\dagger}:=: a_{1}^{\dagger} a_{1}:$ and $: a_{1} a_{1:}^{\dagger}:=a_{1}^{\dagger} a_{1}:$. 
For one- and two-mode combination squeezed vacuum state $V|00\rangle$, its Wigner function is given by

$$
W\left(q_{1}, p_{1} ; q_{2}, p_{2}\right)=\operatorname{tr}\left[V|00\rangle\langle 00| V^{-1} \Delta_{1}\left(q_{1}, p_{1}\right) \Delta_{2}\left(q_{2}, p_{2}\right)\right]=\langle 00|U| 00\rangle,
$$

where $U=V^{-1} \Delta_{1}\left(q_{1}, p_{1}\right) \Delta_{2}\left(q_{2}, p_{2}\right) V$. Further using Eq.(24), noticing that the Weyl ordering has a remarkable property, i.e., the order-invariance of Weyl ordered operators under similar transformations [17-19, which means

$$
V^{-1}:(\circ \circ \circ):{ }^{:} V=: V^{-1}(\circ \circ \circ) V^{:},
$$

as if the "fence" : : : did not exist, thus $U$ can be cast into the following form (see appendix A),

$$
\begin{aligned}
U & =V^{-1}: \delta\left(q_{1}-Q_{1}\right) \delta\left(p_{1}-P_{1}\right) \delta\left(q_{2}-Q_{2}\right) \delta\left(p_{2}-P_{2}\right){ }^{:} V \\
& =\Delta_{1}\left(q_{1} \cosh \lambda-q_{2} e^{-\gamma} \sinh \lambda, p_{1} \cosh \lambda+p_{2} e^{\gamma} \sinh \lambda\right) \\
& \times \Delta_{2}\left(q_{2} \cosh \lambda-q_{1} e^{\gamma} \sinh \lambda, p_{2} \cosh \lambda+p_{1} e^{-\gamma} \sinh \lambda\right) .
\end{aligned}
$$

So the Wigner function of $V|00\rangle$ is given by

$$
W\left(q_{1}, p_{1} ; q_{2}, p_{2}\right)=\frac{1}{\pi^{2}} \exp \left\{-m_{1}\left(q_{1}^{2}+p_{2}^{2}\right)-m_{2}\left(p_{1}^{2}+q_{2}^{2}\right)+2\left(q_{1} q_{2}-p_{1} p_{2}\right) m_{3}\right\},
$$

where

$$
m_{1}=\cosh ^{2} \lambda+e^{2 \gamma} \sinh ^{2} \lambda, m_{2}=\cosh ^{2} \lambda+e^{-2 \gamma} \sinh ^{2} \lambda, m_{3}=\cosh \gamma \sinh 2 \lambda .
$$

In particular, when $\gamma=0$, Eq.(29) becomes

$$
\begin{aligned}
W\left(q_{1}, p_{1} ; q_{2}, p_{2}\right) & =\frac{1}{\pi^{2}} \exp \left\{-\left(p_{1}^{2}+p_{2}^{2}+q_{1}^{2}+q_{2}^{2}\right) \cosh 2 \lambda\right. \\
& \left.+2\left(q_{1} q_{2}-p_{1} p_{2}\right) \sinh 2 \lambda\right\}
\end{aligned}
$$

which is just the Wigner function of the usual TSVS.

\section{Entanglement properties of $V|00\rangle$}

In this section, we consider the entanglement properties of $V|00\rangle$. It is well known that a two-mode Gaussian state can be completely characterized by its first and second statistical moments and the covariance matrix of elements $\sigma$. In general, the first statistical moments can be adjusted by local displacements without affecting entanglement, thus they they will can be set to be zero without loss of generality and the behavior of the covariance matrix $\sigma$ is all important for the study of entanglement. There are several quantitative measurements of quantum entanglement proposed [20 22]. For a two-mode Gaussian state, the entanglement is best characterized by the logarithmic negativity $E_{\mathcal{N}}$, a quantity evaluated in terms of the symplectic eigenvalues of $\sigma$ [23, 24].

In order to evaluate the entanglement of $V|00\rangle$, we reform Eq.(29) as follows in terms of phase space quadrature variables, 


$$
W\left(q_{1}, p_{1} ; q_{2}, p_{2}\right)=\frac{1}{\pi^{2}} \exp \left[-\frac{1}{2}\left(\begin{array}{llll}
q_{1} & p_{1} & q_{2} & p_{2}
\end{array}\right) \sigma^{-1}\left(\begin{array}{llll}
q_{1} & p_{1} & q_{2} & p_{2}
\end{array}\right)^{T}\right],
$$

where the covariance matrix $\sigma$ of this OTCSS is [25]

$$
\sigma=\left(\begin{array}{cc}
u & w \\
w^{T} & v
\end{array}\right), u=\frac{1}{2}\left(\begin{array}{cc}
m_{2} & 0 \\
0 & m_{1}
\end{array}\right), v=\frac{1}{2}\left(\begin{array}{cc}
m_{1} & 0 \\
0 & m_{2}
\end{array}\right), w=\frac{1}{2}\left(\begin{array}{cc}
m_{3} & 0 \\
0 & -m_{3}
\end{array}\right) .
$$

In particular, when $\gamma=0, m_{1}=m_{2}=\cosh 2 \lambda, m_{3}=\sinh 2 \lambda$, Eq.(32) just reduces to the so-called standard form of covariance matrix for TSVS [24].

The condition for entanglement of a Gaussian state is derived from the partially transposed density matrix (PPT criterion) 24], according to the smallest symplectic eigenvalue $\tilde{n}_{s}$ of the partially transposed state, $\tilde{n}_{s}<\frac{1}{2}$, i.e., $\tilde{n}_{s} \geqslant \frac{1}{2}$ means the a two-mode Gaussian state is separable, where $\tilde{n}_{s}$ is defined as

$$
\tilde{n}_{s}=\min \left[\tilde{n}_{+}, \tilde{n}_{-}\right]
$$

and $\tilde{n}_{ \pm}$is given by [26]

$$
\tilde{n}_{ \pm}=\sqrt{\frac{\tilde{\Delta}(\sigma) \pm\left(\tilde{\Delta}(\sigma)^{2}-4 \operatorname{det} \sigma\right)^{1 / 2}}{2}}
$$

where $\tilde{\Delta}(\sigma)=\Delta(\tilde{\sigma})=\operatorname{det} u+\operatorname{det} v-2 \operatorname{det} w$.

Using Eqs.(32)-(34), the corresponding sympletic eigenvalues $\tilde{n}_{ \pm}$are then given by

$$
\tilde{n}_{ \pm}=\frac{1}{2}\left(\sqrt{m_{1} m_{2}} \pm m_{3}\right)
$$

One the other hand, the corresponding quantification of entanglement is given by the logarithmic negativity $E_{\mathcal{N}}$ defined as [20,26, 27],

$$
E_{\mathcal{N}}=\max \left[0,-\ln 2 \tilde{n}_{s}\right] .
$$

From Eqs.(32), (35) and (36), one can clearly see that the logarithmic negativity $E_{\mathcal{N}}$ is dependent on $\lambda$ and $\gamma$. In figure 1, we plot the logarithmic negativity $E_{\mathcal{N}}$ as a function of parameters $\lambda$ and $\gamma$. From Fig.1, we clearly see a new feature, i.e., in presence of parameter $\gamma$, the logarithmic negativity becomes larger than that of tha usual squeezed state $(\gamma=0)$.

\section{Violations of Bell's inequality for $V|00\rangle$}

We now turn our attention to the nonlocal properties of $V|00\rangle$ in terms of the Bell's inequality. For a two-mode continuous variable system, the Bell's inequality is given, using correlations between parity measurement, by

$$
|\mathcal{B}| \equiv\left|\left\langle\hat{\Pi}^{a b}\left(\alpha^{\prime}, \beta^{\prime}\right)+\hat{\Pi}^{a b}\left(\alpha, \beta^{\prime}\right)+\hat{\Pi}^{a b}\left(\alpha^{\prime}, \beta\right)-\hat{\Pi}^{a b}(\alpha, \beta)\right\rangle\right| \leqslant 2,
$$




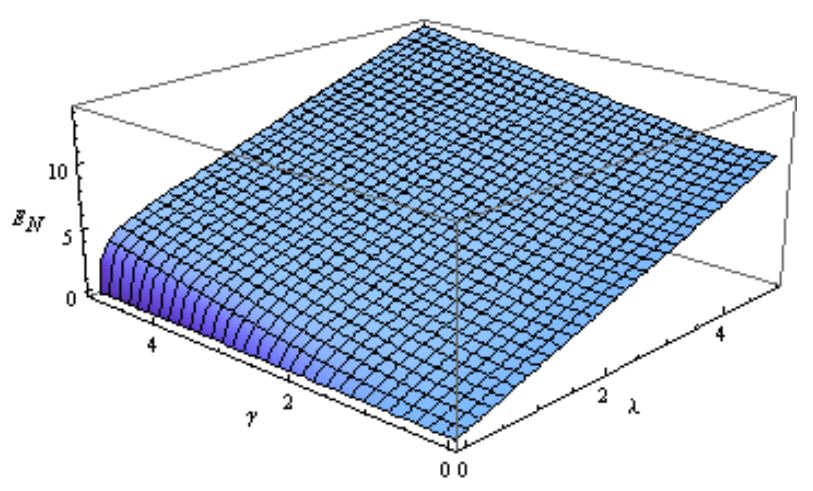

Figure 1: (Color online) The logarithmic negativity $E_{\mathcal{N}}$ as a function of parameters $\lambda$ and $\gamma$.

where $B$ is the Bell function, and the superscripts $a$ and $b$ denote the modes and $\hat{\Pi}^{a b}(\alpha, \beta)$ is the displaced parity operator (Wigner operator) [28] defined as

$$
\begin{aligned}
\hat{\Pi}^{a b}(\alpha, \beta) & \equiv \hat{\Pi}^{a}(\alpha) \hat{\Pi}^{b}(\beta)=D(\alpha) D(\beta)(-1)^{a^{\dagger} a+b^{\dagger} b} D^{\dagger}(\beta) D^{\dagger}(\alpha) \\
& =\pi^{2} \Delta_{a}(\alpha) \Delta_{b}(\beta),
\end{aligned}
$$

where $\alpha=\left(q_{1}+i p_{1}\right) / \sqrt{2}, \beta=\left(q_{2}+i p_{2}\right) / \sqrt{2}$. The expectation value of this displaced parity operator is just proportional to the two-mode Wigner function, i.e.,

$$
\Pi(\alpha, \beta)=\operatorname{tr}\left[\rho \hat{\Pi}^{a b}(\alpha, \beta)\right]=\pi^{2} W(\alpha, \beta),
$$

which shows that the connection between this displaced parity operator and Wigner function provides an equivalent definition [29].

The Bell function is measured for any of four combinations of $\alpha=0, \sqrt{J} e^{i \varphi}$ and $\beta=$ $0, \sqrt{J} e^{i \theta}$, where $J\left(=|\alpha|^{2}=|\beta|^{2}\right)$ is a positive constant characterizing the magnitude of the displacement. From these quantities we construct the combination [30]

$$
\mathcal{B} \equiv \pi^{2}\left[W(0,0)+W\left(\sqrt{J} e^{i \varphi}, 0\right)+W\left(0, \sqrt{J} e^{i \theta}\right)-W\left(\sqrt{J} e^{i \varphi}, \sqrt{J} e^{i \theta}\right)\right] .
$$

In particular, when $\varphi=0, \theta=\pi$, Eq.(40) just reduces to Eq.(7) in Ref. [28]. Then we can test Bell's inequality $-2 \leqslant \mathcal{B} \leqslant 2$ by means of the two-mode Wigner function measurement. Recently, a generalized quasiprobability function is proposed to test quantum nonlocality [31, which includes two-type of Bell-inequality by using the Wigner function [32] and the $Q$-function 30 as its limiting cases.

By noticing that $\alpha=\left(q_{1}+i p_{1}\right) / \sqrt{2}, \beta=\left(q_{2}+i p_{2}\right) / \sqrt{2}$, and $\left(\begin{array}{cccc}q_{1} & p_{1} & q_{2} & p_{2}\end{array}\right) N^{-1}=$

$$
\begin{aligned}
& \left(\begin{array}{llll}
\alpha^{*} & \alpha & \beta^{*} & \beta
\end{array}\right), N=\frac{1}{\sqrt{2}}\left(\begin{array}{cccc}
1 & i & 0 & 0 \\
1 & -i & 0 & 0 \\
0 & 0 & 1 & i \\
0 & 0 & 1 & -i
\end{array}\right) \text {, we can put Eq.(31) into another form } \\
& W(\alpha ; \beta)=\frac{1}{\pi^{2}} \exp \left[-\frac{1}{2}\left(\begin{array}{llll}
\alpha^{*} & \alpha & \beta^{*} & \beta
\end{array}\right) M\left(\begin{array}{llll}
\alpha^{*} & \alpha & \beta^{*} & \beta
\end{array}\right)^{T}\right],
\end{aligned}
$$




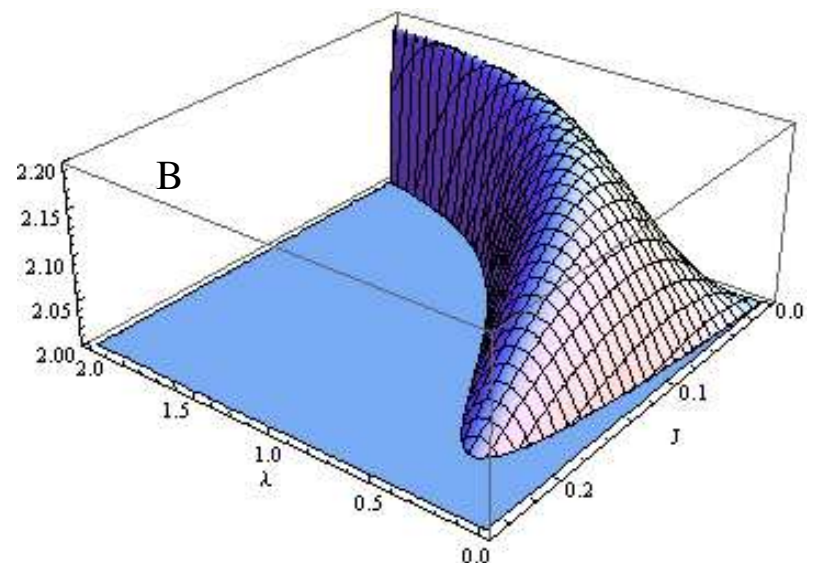

Figure 2: (Color online) Plot of the Bell function $\mathcal{B}$ as a function of parameters $\lambda$ and $J$, for $\gamma=0, \theta=\pi, \varphi=0$. Only values exceeding the bound imposed by local theories are shown.

where $\bar{M}$ is a $4 \times 4$ Hermitian matrix

$$
M=\left(\begin{array}{cccc}
m_{1}-m_{2} & m_{1}+m_{2} & -2 m_{3} & 0 \\
m_{1}+m_{2} & m_{1}-m_{2} & 0 & -2 m_{3} \\
-2 m_{3} & 0 & m_{2}-m_{1} & m_{1}+m_{2} \\
0 & -2 m_{3} & m_{1}+m_{2} & m_{2}-m_{1}
\end{array}\right)
$$

Substituting Eq.(41) into Eq.(40) we have

$$
\begin{aligned}
\mathcal{B}=1 & +\exp \left[-2 J \cosh ^{2} \lambda-2 J\left(e^{2 \gamma} \cos ^{2} \varphi+e^{-2 \gamma} \sin ^{2} \varphi\right) \sinh ^{2} \lambda\right] \\
& +\exp \left[-2 J \cosh ^{2} \lambda-2 J\left(e^{2 \gamma} \sin ^{2} \theta+e^{-2 \gamma} \cos ^{2} \theta\right) \sinh ^{2} \lambda\right] \\
& -\exp \left\{-4 J \cosh ^{2} \lambda-2 J\left(\cos ^{2} \varphi+\sin ^{2} \theta\right) e^{2 \gamma} \sinh ^{2} \lambda\right. \\
& \left.-2 J\left(\sin ^{2} \varphi+\cos ^{2} \theta\right) e^{-2 \gamma} \sinh ^{2} \lambda+4 J \cos (\theta+\varphi) \cosh \gamma \sinh 2 \lambda\right\},
\end{aligned}
$$

Thus we can say that $V|00\rangle$ is quantum mechanically nonlocal as $|\mathcal{B}|>2$, and the nonlocality is stronger with the increase of $|\mathcal{B}|$. From Eq.(43) one can see that the degree of nonlocality not only depends on the coherent amplitude $J$, on the phases $\theta$ and $\varphi$, but also on the parameter $\gamma$. In particular, when $\varphi=0, \theta=\pi$, Eq.(43) just reduces to

$$
\begin{aligned}
\mathcal{B}=1 & +\exp \left[-2 J\left(\cosh ^{2} \lambda+e^{2 \gamma} \sinh ^{2} \lambda\right)\right] \\
& +\exp \left[-2 J\left(\cosh ^{2} \lambda+e^{-2 \gamma} \sinh ^{2} \lambda\right)\right] \\
& -\exp \left[-4 J\left(\cosh ^{2} \lambda+\cosh 2 \gamma \sinh ^{2} \lambda\right)-4 J \cosh \gamma \sinh 2 \lambda\right],
\end{aligned}
$$

which further becomes Eq.(7) in Ref. 28 with $\gamma=0$.

In figure 2, we plot Bell function in the space spanned by parameters $J$ and $\lambda$ with $\gamma=0$ (corresponding to the usual squeezed vacuum state). From Fig. 2, one can clearly see that the result (43) violates the upper bound imposed by local theories. With the increase of $\lambda$, the violation of Bell's inequality becomes more observable for smaller $J$.

As depicted in Fig. 3, the Bell function also violates the upper bound in the space spanned by parameters $\gamma$ and $\lambda$ with given $\alpha, \beta$ values. From Fig.3, one can see that for a given small $\gamma$, the violation of Bell's inequality becomes more observable with the increase 


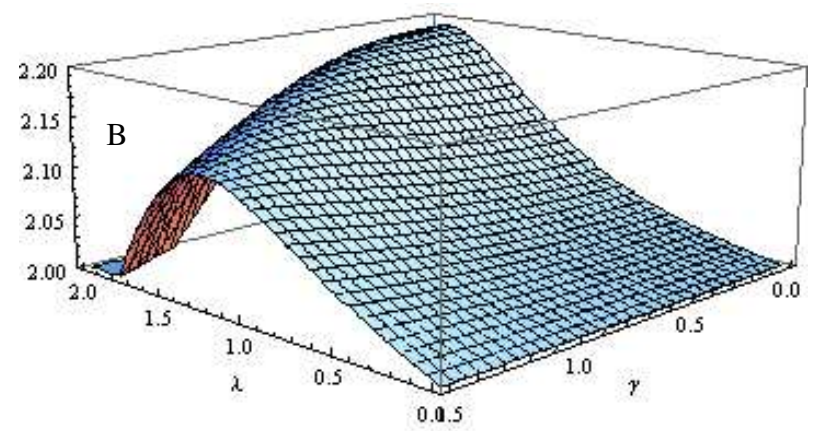

Figure 3: (Color online) Plot of the Bell function $\mathcal{B}$ as a function of parameters $\lambda$ and $\gamma$,for given $|\alpha|=|\beta|=0.05$ and $\theta=\pi, \varphi=0$. Only values exceeding the bound imposed by local theories are shown.

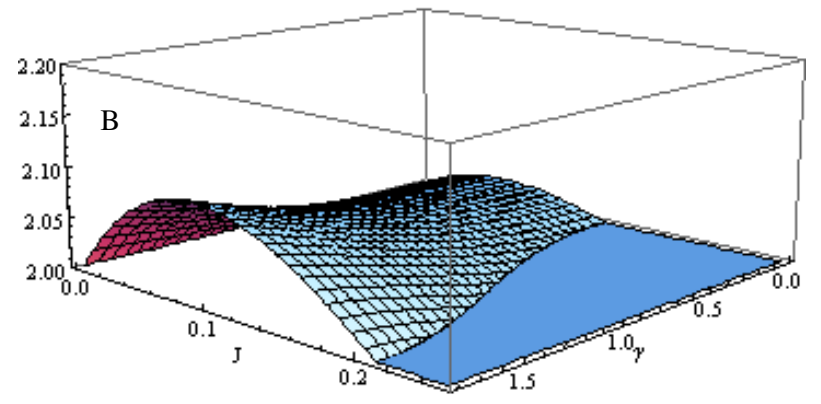

Figure 4: (Color online) Plot of the Bell function $\mathcal{B}$ as a function of parameters $J$ and $\gamma$,for given $\theta=\pi, \varphi=0$, and $\lambda=0.1$. Only values exceeding the bound imposed by local theories are shown. 

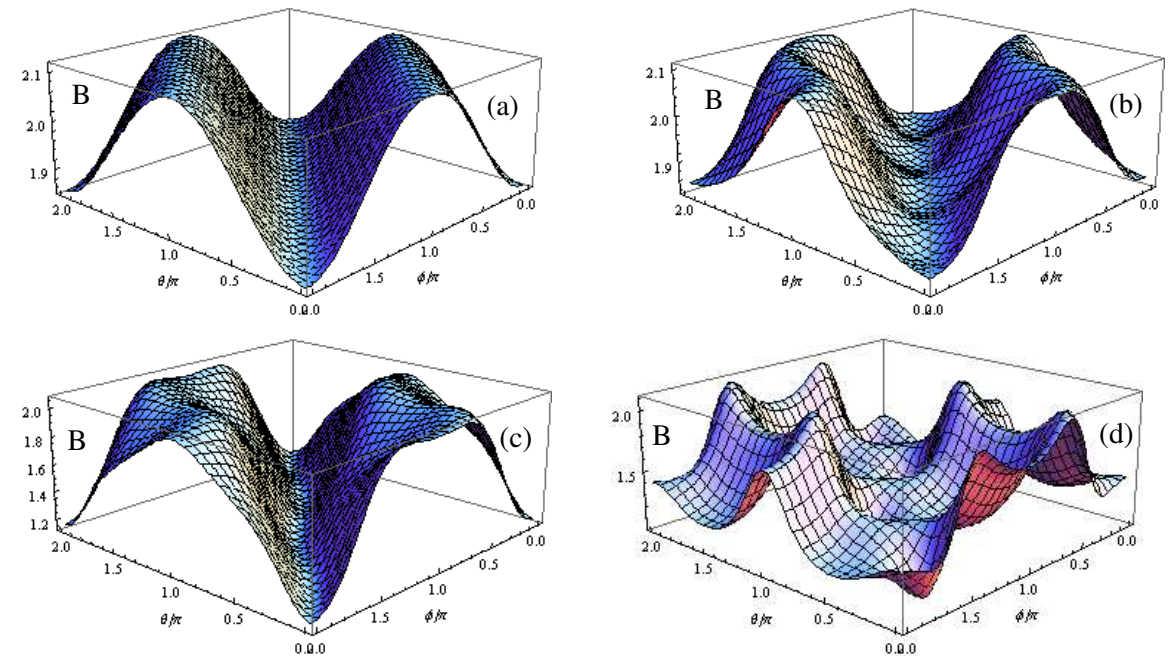

Figure 5: (Color online) Plot of the Bell function $\mathcal{B}$ as a function of parameters $\theta$ and $\varphi$,for given $J=0.01$, and (a) $\lambda=0.5, \gamma=1$; (b) $\lambda=0.5, \gamma=2$;(c) $\lambda=1, \gamma=1$;(a) $\lambda=1, \gamma=2$.

of $\lambda$; while for a large $\gamma$, the Bell function is not always monotone for an increasing $\lambda$; In a certain smaller regime of $\lambda$, it is found that the violation of Bell's inequality becomes more observable with increasing $\gamma$, which indicates that the nonlocality of $V|00\rangle$ is enhanced due to the presence of $\gamma$ (also see Fig.4). In addition, for a certain larger regime of $\lambda$, the maximum value of $\mathcal{B}$ becomes smaller with the increase of $\gamma$.

On the other hand, from the expression we see that the degree of nonlocality depends on the coherent amplitude $J$, and on the squeezed parameters $\lambda$ and $\gamma$, and on the phases $\varphi, \theta$. We have plotted the Bell function $\mathcal{B}$ as a function of the phases $\varphi, \theta$ and with fixed $J=0.01$ and several different $\lambda, \gamma$, as shown in Fig. 5. One can clearly see from Fig. 5 that $\mathcal{B}$ is always greater than zero and for a smaller $\lambda$, the variable $\mathcal{B}$ reaches its maxmum value for $\varphi=0, \theta=\pi$ or $\varphi=\pi, \theta=0$ (see Fig.5(a),(b)); while for a larger $\lambda$, the phases $\varphi, \theta$ corresponding to the maxmum value of $\mathcal{B}$ are different from those above and vary as $\gamma$ parameter.

\section{Application to quantum teleportation with $V|00\rangle$}

In quantum teleportation (QT), an unknown state is transmitted from a sender (Alice) to a receiver (Bob) via a quantum channel with the aid of some classical information. This process may be regarded as sending and extracting quantum information via the quantum channel. QT was firstly proposed by Barnnett et al in the discrete variable regime [33] followed by experimental demonstration [34,35]. For the continuous variables (CVs) case, the theoretical analysis of teleportation was firstly made by Vaidman [36. The role of teleportation in the CV quantum information is analyzed in the review Ref. [37.

Recently, a CV teleportation protocol has been given in terms of the characteristic functions (CFs) of the quantum states involved (input, source and teleported (output) states) [38. By using the Weyl expansion of density operator, it is shown that the CF $\chi_{\text {out }}(\beta)$ of the output state has a remarkably factorized form

$$
\chi_{\text {out }}(\beta)=\chi_{\text {in }}(\beta) \chi_{E}\left(\beta^{*}, \beta\right),
$$


where $\chi_{i n}(\beta)$ and $\chi_{E}\left(\beta^{*}, \beta\right)$ are the CFs of the input state and the entangled source, respectively, $\chi_{i n}\left(\alpha_{1}\right)=\operatorname{tr}\left[D_{1}\left(\alpha_{1}\right) \rho\right]$ and $\chi_{E}\left(\alpha_{1}, \alpha_{2}\right)=\operatorname{tr}\left[D_{1}\left(\alpha_{1}\right) D_{2}\left(\alpha_{2}\right) \rho\right], D_{i}\left(\alpha_{i}\right)$ is the displacement operator corresponding to mode $i$, and $\rho$ is the density operator associated to the state.

In order to measure the success probability of a teleportation protocol, it is convenient to use the fidelity of teleportation $\mathcal{F}=\operatorname{tr}\left(\rho_{\text {in }} \rho_{\text {out }}\right)$, an overlap between the input state $\rho_{\text {in }}$ and the output (teleported) state $\rho_{\text {out }}$, which can, in the CF form, be expressed as

$$
\mathcal{F}=\int \frac{d^{2} \eta}{\pi} \chi_{\text {in }}(\eta) \chi_{\text {out }}(-\eta)
$$

Substituting Eq.(45) into (46) yields

$$
\mathcal{F}=\int \frac{d^{2} \eta}{\pi}\left|\chi_{i n}(\eta)\right|^{2} \chi_{E}\left(-\eta^{*},-\eta\right)
$$

In the following we use Eq. (47) to analyze the efficiency of teleportation for $V|00\rangle$ as a quantum channel.

Let us first consider Braunstein and Kimble protocol [39] of QT for single-mode coherent states $|\beta\rangle$, whose $\mathrm{CF}$ reads

$$
\chi_{\mathrm{coh}}(\alpha)=\exp \left[-\frac{1}{2}|\alpha|^{2}+\alpha \beta^{*}-\alpha^{*} \beta\right] .
$$

For the OTCSS, its CF is given by (see Appendix B)

$$
\chi(\alpha ; \beta)=\exp \left[-\frac{1}{2}\left(\begin{array}{llll}
\alpha^{*} & \alpha & \beta^{*} & \beta
\end{array}\right) \frac{1}{4} M\left(\begin{array}{llll}
\alpha^{*} & \alpha & \beta^{*} & \beta
\end{array}\right)^{T}\right],
$$

where $M$ is defined in Eq.(42). Upon substituting Eqs.(48) and (49) into (47), we worked out the fidelity for teleporting a coherent state based on the OTCSS (19),

$$
\mathcal{F}=\frac{1}{1-f}
$$

where $f=\cosh \gamma \sinh 2 \lambda-\cosh ^{2} \lambda-\cosh 2 \gamma \sinh ^{2} \lambda$. Eq.(150) indicates that the fidelity is only dependent on the parameters $\lambda$ and $\gamma$, and is independent of amplitude of the coherent state. In particular, when $\gamma=0$, Eq.(50) just reduces to $\mathcal{F}=(1+\tanh \lambda) / 2$ [40].

Next we consider to teleport the single-mode squeezed vacuum state, $\exp \left[r / 2\left(a^{2}-a^{\dagger 2}\right)\right]|0\rangle$, whose CF reads

$$
\chi_{s q}(\alpha)=\exp \left[-\frac{1}{2}|\alpha|^{2} \cosh 2 r-\frac{1}{4}\left(\alpha^{2}+\alpha^{* 2}\right) \sinh 2 r\right],
$$

substituting Eqs.(51),(49) into Eq.(47) yields the fidelity,

$$
\mathcal{F}(r)=\frac{1}{\sqrt{f^{2}-2 f \cosh 2 r+1}} .
$$

In order to compare the fidelity obtained by the TSVS channel and the OTCSS channel, we plot figure for the difference fidelity $(\mathcal{F}(r)-\mathcal{F}(0))$ as a function of parameters $(\lambda$ and $\gamma$ ) in Fig.6. From Fig.6, one can see that there is a region spanned by $\lambda$ and $\gamma$ in which the fidelity of OTCSS channel is larger than the other one and the difference fidelity becomes smaller with the increase of $r$. 

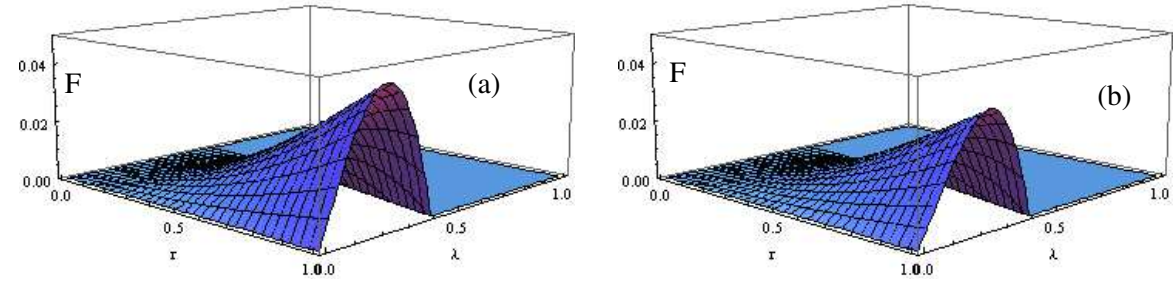

Figure 6: (Color online) Plot of the fidelity $\mathcal{F}$ as a function of parameters $\lambda$ and $\gamma$,(a) the initial coherent state, with $r=0$;(b) the initial squeezed vacuum state with $r=1$.

\section{Conclusion}

In conclusion, we have introduced a one- and two-mode combination squeezed state (OTCSS) which can be considered as a generalized two-mode squeezed state and investigated the entanglement properties and quantum noncocality of this state in terms of logarithmic negativity and the Bell's inequality, respectively. It is shown that this state presents larger entanglement than that of the usual two-mode squeezed vacuum state (TSVS); In a certain smaller regime of $\lambda$, it is found that the violation of Bell's inequality becomes more observable with increasing $\gamma$, which indicates that the nonlocality of $V|00\rangle$ can be stronger than that of TSVS due to the presence of $\gamma$. In addition, application to quantum teleportation with OTCSS is also considered, which shows that there is a region spanned by $\lambda$ and $\gamma$ in which the fidelity of OTCSS channel is larger than that of TSVS.

ACKNOWLEDGEMENT: Work supported by a grant from the Key Programs Foundation of Ministry of Education of China (No. ) and the Research Foundation of the Education Department of Jiangxi Province of China (No. GJJ10097).

\section{Appendix A: Derivation of Eq.(29)}

Using the order-invariance of Weyl ordered operators under similar transformations (27) and Eqs.(9)-(12), we have

$$
\begin{aligned}
& U=V^{-1}: \delta\left(q_{1}-Q_{1}\right) \delta\left(p_{1}-P_{1}\right) \delta\left(q_{2}-Q_{2}\right) \delta\left(p_{2}-P_{2}\right){ }^{:}: \\
& ={ }_{:}^{:} \delta\left(q_{1}-Q_{1} \cosh \lambda-Q_{2} e^{-\gamma} \sinh \lambda\right) \delta\left(q_{2}-Q_{2} \cosh \lambda-Q_{1} e^{\gamma} \sinh \lambda\right) \\
& \times \delta\left(p_{1}-P_{1} \cosh \lambda+P_{2} e^{\gamma} \sinh \lambda\right) \delta\left(p_{2}-P_{2} \cosh \lambda+P_{1} e^{-\gamma} \sinh \lambda\right): \\
& =: \delta\left(\left(\begin{array}{l}
q_{1} \\
q_{2}
\end{array}\right)-\left(\begin{array}{cc}
\cosh \lambda & e^{-\gamma} \sinh \lambda \\
e^{\gamma} \sinh \lambda & \cosh \lambda
\end{array}\right)\left(\begin{array}{l}
Q_{1} \\
Q_{2}
\end{array}\right)\right) \\
& \times \delta\left(\left(\begin{array}{l}
p_{1} \\
p_{2}
\end{array}\right)-\left(\begin{array}{cc}
\cosh \lambda & -e^{\gamma} \sinh \lambda \\
-e^{-\gamma} \sinh \lambda & \cosh \lambda
\end{array}\right)\left(\begin{array}{c}
P_{1} \\
P_{2}
\end{array}\right)\right): \\
& =: \delta\left(\left(\begin{array}{cc}
\cosh \lambda & -e^{-\gamma} \sinh \lambda \\
-e^{\gamma} \sinh \lambda & \cosh \lambda
\end{array}\right)\left(\begin{array}{l}
q_{1} \\
q_{2}
\end{array}\right)-\left(\begin{array}{l}
Q_{1} \\
Q_{2}
\end{array}\right)\right) \\
& \times \delta\left(\left(\begin{array}{cc}
\cosh \lambda & e^{\gamma} \sinh \lambda \\
e^{-\gamma} \sinh \lambda & \cosh \lambda
\end{array}\right)\left(\begin{array}{l}
p_{1} \\
p_{2}
\end{array}\right)-\left(\begin{array}{c}
P_{1} \\
P_{2}
\end{array}\right)\right):
\end{aligned}
$$


which indicates that (comparing with Eq.(24))

$$
\begin{aligned}
U & ={ }^{:} \delta\left(q_{1} \cosh \lambda-q_{2} e^{-\gamma} \sinh \lambda-Q_{1}\right) \delta\left(p_{1} \cosh \lambda+p_{2} e^{\gamma} \sinh \lambda-P_{1}\right) \\
& \times \delta\left(q_{2} \cosh \lambda-q_{1} e^{\gamma} \sinh \lambda-Q_{2}\right) \delta\left(p_{2} \cosh \lambda+p_{1} e^{-\gamma} \sinh \lambda-P_{2}\right): \\
& =\text { Eq. (29). }
\end{aligned}
$$

Thus the Wigner function of $V|00\rangle$ is

$$
\begin{aligned}
W\left(q_{1}, p_{1} ; q_{2}, p_{2}\right) & =\frac{1}{\pi^{2}} \exp \left\{-\left(q_{1} \cosh \lambda-q_{2} e^{-\gamma} \sinh \lambda\right)^{2}-\left(q_{2} \cosh \lambda-q_{1} e^{\gamma} \sinh \lambda\right)^{2}\right. \\
& \left.-\left(p_{1} \cosh \lambda+p_{2} e^{\gamma} \sinh \lambda\right)^{2}-\left(p_{2} \cosh \lambda+p_{1} e^{-\gamma} \sinh \lambda\right)^{2}\right\} \\
& =(29) .
\end{aligned}
$$

\section{Appendix B: Derivation of characteristic function of $V|00\rangle$}

For two-mode quantum state $V|00\rangle$, its characteristic function is given by

$$
\begin{aligned}
\chi\left(q_{1}, p_{1} ; q_{2}, p_{2}\right) & =\operatorname{tr}\left[V|00\rangle\langle 00| V^{-1} D_{1}\left(q_{1}, p_{1}\right) D_{2}\left(q_{2}, p_{2}\right)\right] \\
& =\left\langle 00\left|V^{-1} D_{1}\left(q_{1}, p_{1}\right) D_{2}\left(q_{2}, p_{2}\right) V\right| 00\right\rangle,
\end{aligned}
$$

where $D_{i}\left(q_{i}, p_{i}\right)(i=1,2)$ are the displacement operators, defined by $D_{i}\left(q_{i}, p_{i}\right)=\exp \left[\mathrm{i}\left(p_{i} Q_{i}-q_{i} P_{i}\right)\right]$.

Noticing that the Weyl ordering of $D_{i}\left(q_{i}, p_{i}\right)$ is itself, $D_{i}\left(q_{i}, p_{i}\right)=: D_{i}\left(q_{i}, p_{i}\right):$, and that a remarkable property of the invariance of Weyl ordered operators under similar transformations (27), we have

$$
\begin{aligned}
& V^{-1} D_{1}\left(q_{1}, p_{1}\right) D_{2}\left(q_{2}, p_{2}\right) V \\
& =V^{-1}: \exp \left[i\left(p_{1} Q_{1}-q_{1} P_{1}\right)\right] \exp \left[i\left(p_{2} Q_{2}-q_{2} P_{2}\right)\right]_{:}^{:} V \\
& =: \exp \left[i\left(p_{1}\left(Q_{1} \cosh \lambda+Q_{2} e^{-\gamma} \sinh \lambda\right)-q_{1}\left(P_{1} \cosh \lambda-P_{2} e^{\gamma} \sinh \lambda\right)\right)\right] \\
& \times \exp \left[i\left(p_{2}\left(Q_{2} \cosh \lambda+Q_{1} e^{\gamma} \sinh \lambda\right)-q_{2}\left(P_{2} \cosh \lambda-P_{1} e^{-\gamma} \sinh \lambda\right)\right)\right]: \\
& =: \exp \left[i\left(p_{1}^{\prime} Q_{1}-q_{1}^{\prime} P_{1}\right)\right] \exp \left[i\left(p_{2}^{\prime} Q_{2}-q_{2}^{\prime} P_{2}\right)\right]: \\
& =D_{1}\left(q_{1}^{\prime}, p_{1}^{\prime}\right) D_{2}\left(q_{2}^{\prime}, p_{2}^{\prime}\right)
\end{aligned}
$$

where

$$
\begin{aligned}
& q_{1}^{\prime}=q_{1} \cosh \lambda-q_{2} e^{-\gamma} \sinh \lambda, p_{1}^{\prime}=p_{1} \cosh \lambda+p_{2} e^{\gamma} \sinh \lambda, \\
& q_{2}^{\prime}=q_{2} \cosh \lambda-q_{1} e^{\gamma} \sinh \lambda, p_{2}^{\prime}=p_{2} \cosh \lambda+p_{1} e^{-\gamma} \sinh \lambda .
\end{aligned}
$$

Then substituting Eqs.(B2), (B3) into Eq.(B1) we can directly obtain the CF of $V|00\rangle$,

$$
\begin{aligned}
\chi\left(q_{1}, p_{1} ; q_{2}, p_{2}\right) & =\left\langle 00\left|D_{1}\left(q_{1}^{\prime}, p_{1}^{\prime}\right) D_{2}\left(q_{2}^{\prime}, p_{2}^{\prime}\right)\right| 00\right\rangle \\
& =\exp \left[-\frac{1}{2}\left(\begin{array}{llll}
q_{1} & p_{1} & q_{2} & p_{2}
\end{array}\right) \sigma\left(\begin{array}{llll}
q_{1} & p_{1} & q_{2} & p_{2}
\end{array}\right)^{T}\right],
\end{aligned}
$$

or

$$
\chi(\alpha ; \beta)=\exp \left[-\frac{1}{2}\left(\begin{array}{llll}
\alpha^{*} & \alpha & \beta^{*} & \beta
\end{array}\right) \bar{\sigma}\left(\begin{array}{llll}
\alpha^{*} & \alpha & \beta^{*} & \beta
\end{array}\right)^{T}\right]
$$


where

$$
\sigma=\frac{1}{2}\left(\begin{array}{cccc}
m_{1} & 0 & -m_{3} & 0 \\
0 & m_{2} & 0 & m_{3} \\
-m_{3} & 0 & m_{2} & 0 \\
0 & m_{3} & 0 & m_{1}
\end{array}\right), \bar{\sigma}=N \sigma N^{T}=\frac{1}{4} M .
$$

When $\gamma=0,\left(m_{1}+m_{2}\right) \rightarrow 2 \cosh 2 \lambda, m_{3} \rightarrow \sinh 2 \lambda$, we have

$$
\chi(\alpha ; \beta)=\exp \left[-\frac{1}{2}\left(|\alpha|^{2}+|\beta|^{2}\right) \cosh 2 \lambda+\frac{1}{2}\left(\alpha^{*} \beta^{*}+\alpha \beta\right) \sinh 2 \lambda\right],
$$

which is just the CF of the usual TSVS.

\section{References}

[1] D. Bouwmeester et al., The Physics of Quantum Information, (Springer, Berlin) 2000.

[2] M. A. Nielsen and I. L. Chuang, Quantum Computation and Quantum Information (Cambridge University Press) 2000.

[3] V. Buzek, J. Mod. Opt. 37 (1990) 303.

[4] R. Loudon, P. L. Knight, J. Mod. Opt. 34 (1987) 709.

[5] V. V. Dodonov, J. Opt. B: Quantum Semiclass. Opt. 4 (2002) R1.

[6] Hong-yi Fan and J. R. Klauder, Phys. Rev. A 49 (1994) 704.

[7] Hong-yi Fan and Y. Fan, Phys. Rev. A 54 (1996) 958.

[8] Li-yun Hu and Hong-yi Fan, Europhys. Lett. 85 (2009) 60001.

[9] A. Einstein, B. Poldolsky and N. Rosen, Phys. Rev. 47 (1935) 777.

[10] Li-yun Hu and Hong-yi Fan, Phys. Rev. A 80 (2009) 022115; Opt. Commun. 282 (2009) 3734 .

[11] Hong-yi Fan and Li-yun Hu, Opt. Commun. 281 (2008) 1629; 281 (2008) 5571.

[12] Hong-yi Fan, J Opt B: Quantum Semiclass. Opt. 5 (2003) R147

[13] Hong-yi Fan, Phys. Rev. A 41 (1990) 1526.

[14] E. P. Wigner, Phys. Rev. 40 (1932) 749.

[15] R. F. O'Connell and E. P. Wigner, Phys. Lett. A 83 (1981) 145.

[16] W. P. Schleich, Quantum Optics in Phase Space, Wiley-VCH, Berlin, 2001.

[17] Hong-yi Fan, J. Phys. A 25 (1992) 3443; Hong-yi Fan, Y. Fan, Int. J. Mod. Phys. A 17 (2002) 701.

[18] Hong-yi Fan, Mod. Phys. Lett. A 15 (2000) 2297.

[19] Hong-yi Fan, Ann. Phys. (New York) 323 (2008) 500; 323 (2008) 1502. 
[20] G. Vidal and R. F. Werner, Phys. Rev. A 65 (2002) 032314.

[21] J. Eisert and M. B. Plenio, J. Mod. Opt. 46 (1999) 145.

[22] S. Virmani and M. B. Plenio, Phys. Lett. A 268 (2000) 31.

[23] L. M. Duan, G. Giedke, J. O. Cirac, and P. Zoller, Phys. Rev. Lett. 84 (2000) 2722.

[24] R. Simon, Phys. Rev. Lett. 84 (2000) 2726.

[25] J. Williamson, Am. J. Math. 58 (1936) 141; V. I. Arnold, Mathematical Methods of Classical Mechanics (Springer-Verlag, New York, 1978).

[26] A. Serafini, F. Illuminati, M. G. A. Paris, and S. De Siena, Phys. Rev. A 69 (2004) 022318 .

[27] G. Adesso, A. Serafini, and F. Illuminati, Phys. Rev. A. 70 (2004) 022318.

[28] K. Banasek and K. Wodkiewicz, Phys. Rev. A. 58 (1998) 4345.

[29] K. Banasek and K. Wodkiewicz, Phys. Rev. Lett. 76 (1996) 4344; S. Wallentowitz and W. Volgel, Phys. Rev. A. 53 (1996) 4528.

[30] J. F. Clauser, M. A. Horne, A. Shimony, and R. A. Holt, Phys. Rev. Lett. 23 (1969) 880.

[31] S-W Lee, H. Jeong, and D. Jaksch, Phys. Rev. A. 80 (2009) 022104.

[32] J. F. Clauser, and M. A. Horne, Phys. Rev. D. 10 (1974) 526.

[33] C. H. Bennett, G. Brassard, C. Crepeau, R. Jozsa, A. Peres, and W. K. Wootters, Phys. Rev. Lett. 70 (1993) 1895.

[34] D. Bouwmeester, J. W. Pan, K. Mattle, M. Eibl, H.Weinfurther, and A. Zeilinger, Nature (London) 390 (1997) 575.

[35] D. Boschi, S. Branca, F. De Martini, L. Hardy, and S. Popescu, Phys. Rev. Lett. 80 (1998) 1121.

[36] L. Vaidmain, Phys. Rev. A 49 (1994) 1473.

[37] S. L. Braunstein and P. van Loock, Rev. Mod. Phys. 77 (2005) 513.

[38] P. Marian and T. A. Marian, Phys. Rev. A. 74 (2006) 042306.

[39] S. L. Braunstein and H. J. Kimble, Phys. Rev. Lett. 80 (1998) 869.

[40] Y. Yang, and F-L. Li, Phys. Rev. A. 80 (2009) 022315. 